\title{
Electronic nose - an instrument for odour nuisances monitoring
}

\author{
Bartosz Szulczyński ${ }^{1, *}$, and Jacek Gębicki ${ }^{1}$ \\ ${ }^{1}$ Department of Process Engineering and Chemical Technology, Faculty of Chemistry, \\ Gdańsk University of Technology, ul. Gabriela Narutowicza 11/12, 80-233 Gdańsk, Poland
}

\begin{abstract}
An increasingly frequent problem of people living in urban agglomerations is the occurrence of odour nuisance. Although the source of these nuisances is different, their common feature is that they are a complex mixture of odour compounds with different odour thresholds. However, from a practical point of view, the most valuable would be a direct link between the odour intensity and the results of on-line analytical air monitoring. Such a possibility is created by the use of electronic noses (devices that are supposed to imitate the human sense of smell) to measure odours. The paper presents the use of an electronic nose combined with multiple liear regression model (MLR) to determine the odour intensity of the two-component mixture samples of commonly known odour compounds: trimethylamine (TMA) and triethylamine (TEA) in concentration range $50-200 \mathrm{ppm} \mathrm{v/v}$. The obtained results were compared with the theoretical values determined using Zwaardemaker and euclidean additivity (EA) models. For high concentrations of substances in

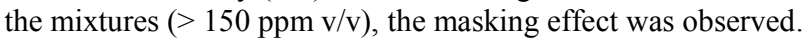

\section{Introduction}

The ability to identify and distinguish odour substances for some time was possible only through the human sense of smell. With the development of science and technology, there is a growing interest in devices constructed and functioning in the image of human senses. Over the last 30 years, analytical systems have been developed that could in some ways replace human sense of smell [1-3]. Devices that have aroused interest due to the use of a wide range of chemical sensors in their construction are electronic noses. These devices, being analogues of the sense of human smell, allow their application in many fields of science and industry, such as: medical diagnostics, environmental protection, food and chemical industry or criminology [4-7]. Electronic noses enable a holistic analysis of the composition of the gas mixture, without the need to separate and identify its individual components $[8,9]$. In comparison to other techniques used to analyze odours such as olfactometry or gas chromatography, electronic noses have additional advantages. In relation to olfactometric techniques, there is a lack of olfactory adaptation phenomenon and having trained personnel with a specific olfactory perception. In relation to

* Corresponding author: bartosz.szulczynski@.pg.edu.pl 
chromatographic techniques, electronic noses are characterized by a short analysis time and a lower price of the device. Electronic noses with their advantages and certain limitations complement the above-mentioned measuring techniques used for odour analysis.

The smell, which is a sensual impression, is relatively difficult to quantitative description. Four basic odour parameters are determined: odour concentration, odour threshold, odour intensity and hedonic tone. However, the values of these parameters determined for mixtures of odour substances are not additive. This is due to the occurrence of the odour interactions based on the mutual masking, strengthening or weakening of odours. Studies on the types of olfactory interactions have been conducted for a very long time, but so far have not led to the explanation of the mechanism of these processes. The objects of experimental research are usually air samples containing only two or three types of odorants $[10,11]$.

The paper presents the use of an electronic nose to determine the odour intensity of the two-component mixture samples of trimethylamine (TMA) and triethylamine (TEA) - two commonly known odour compounds. The results were compared with the theoretically determined values.

\subsection{Odour intensity}

The odour intensity is the intensity of the smell, i.e. the strength of the olfactory sensation, which is associated with the frequency of nerve impulses in a given olfactory receptor. The intensity depends on the amount of the odorants, which are inhaled with air. They come into contact with olfactory chemoreceptors, so the intensity depends on the concentration of the compound in the inhaled air. The most frequently used mathematical equations describe the dependence of the odour intensity on the concentration of odorants. They are the expression of psychophysical rights of a general nature and concern the perception of all sensory impressions. Two equations are commonly used to describe the odour intensity: Weber-Fechner law (1) and Stevens's power law (2).

$$
\begin{gathered}
I=k_{W-F} \cdot \log \frac{c}{c_{O T}} \\
I=k_{S} \cdot c^{n}
\end{gathered}
$$

where: $I$ - odour intensity; $k_{W-F}, k_{S}$ - equation coefficients; $c$ - compound concentration; $c_{O T}$ - odour threshold, $n$ - empirical constant value.

Equations (1) and (2) are applicable to individual compound concentration. The combination of experimental values for pure chemical compounds does not allow to predict the intensity of their mixtures. So it is not an additive amount. This is due to odour interactions occurring in the odorant mixture, which contribute to strengthening, weakening or masking one fragrance with the other [12].

In the case of mixtures, mathematical models are used that allow to estimate the odour intensity of the mixture using intensity values for its individual components. One of these models is the Zwaardemaker model. It is often called the vector summation model. This takes on form (3) for a two-component mixture and form (4) for a three-component mixture. The coefficient $\alpha$ found in the equations (3) and (4) is approximately constant for one pair of mixture components.

$$
\begin{gathered}
I_{A B}=\sqrt{I_{A}^{2}+I_{B}^{2}+2 \cdot I_{A} I_{B} \cdot \cos \alpha_{A B}} \\
I_{A B C}=\sqrt{I_{A}^{2}+I_{B}^{2}+I_{C}^{2}+2 \cdot I_{A} I_{B} \cdot \cos \alpha_{A B}+2 \cdot I_{B} I_{C} \cdot \cos \alpha_{B C}+2 \cdot I_{A} I_{C} \cdot \cos \alpha_{A C}}
\end{gathered}
$$


Recently, studies on the determination of this coefficient for compounds from the group of aldehydes, esters or aromatic hydrocarbons were carried out by researchers who determined its value at the level of: $0.1,0.2$ and 0.3 respectively [13].

Another model used to estimate the odour intensity is the EA model (Euclidean Additivity). The intensity of the mixture is determined using equation (5). This is a special case of the Zwaardemaker model with the $\cos \alpha=0$ value.

$$
I_{A B}=\sqrt{I_{A}^{2}+I_{B}^{2}}
$$

\subsection{Electronic nose}

Active development of industry is inevitably connected with the emission of chemical compounds to the atmosphere. Taking into account standards and legal regulations regarding the emission of dangerous compounds into the environment, innovative technologies have been developed to identify and determine the concentrations of odorous volatile compounds [14]. In order to provide a more precise and objective assessment of the odorous compounds than in the case of sensory analysis, an electronic nose was constructed using chemical sensors. These sensors initially appeared individually, but with the development of technology began to create special gas sensor matrices, which are equipped with several, or even more, gas chemical sensors [15].

The electronic nose was first developed in 1982 by Dodd and Persuad to imitate the sense of human sense of smell. The device was designed mainly to detect volatile chemical compounds emitted from various sources. An electronic nose (e-nose) is structurally constructed from four blocks: sampling, detection, data processing and pattern recognition system (Figure 1).

\section{ELECTRONIC NOSE SYSTEMS}

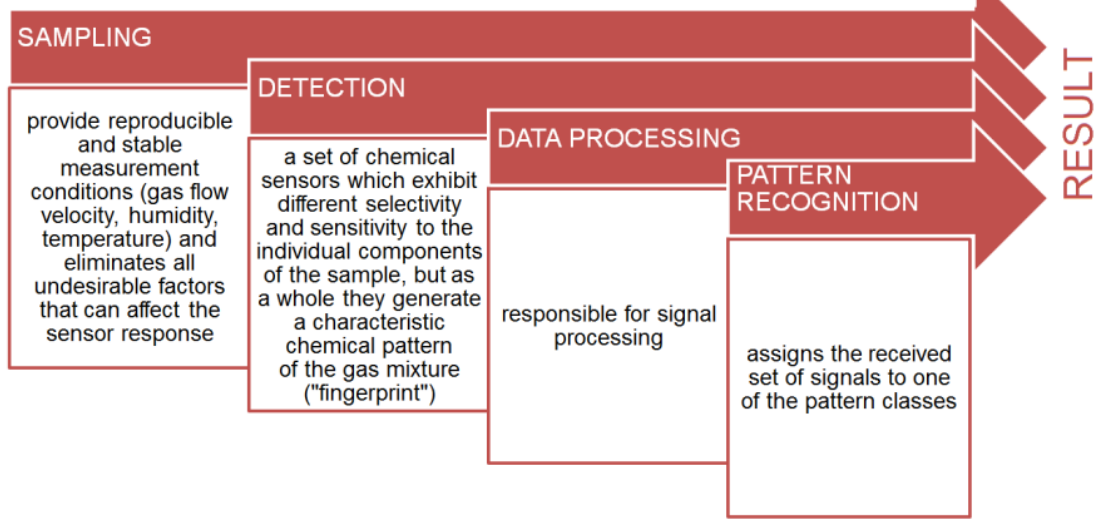

Fig. 1. Electronic nose systems.

The concept of an electronic nose is collinear with a human sense of smell. Its operation is based on the use of sensors matrix, which after exposure to odours generate a proper set of signals. The sampling system has the task of transferring volatile molecules from the atmosphere to the sensors array that are enclosed in the chamber under constant temperature and humidity conditions. In the detection zone, chemical gas sensors are located. They convert the chemical information into an analytically useful electric signal. The most common sensors used for building electronic noses are: electrochemical sensors (EC), semiconductor sensors (MOS), conductivity sensors with a layer of conductive 
polymers (CP), surface acoustic wave (SAW) sensors, piezoelectric sensors (QMB) and photoionization sensors (PID) [9].

The determination of odour intensity using an electronic nose requires the use of calibration models. To develop such models, a set of explanatory variables is used (signals from electronic nose sensors) and a set of dependent variables (values of the odour intensity expressed in the verbal scale). One of the most popular calibration techniques are: multiparameter linear regression (MLR), principle component regression (PCR), partial least squares regression (PLS) and artificial neural networks (ANN). These methods have been successfully applied to monitor changes in the aroma concentration in such processes as: biofiltration or wastewater treatment as well as to support measurements made using dynamic olfactometry or sensory analysis [16-23].

The presented research uses a self-designed and constructed prototype of an electronic nose equipped with eight chemical sensors: 1 PID sensor, 2 electrochemical sensors and 5 semiconductor sensors. The models of the sensors used are shown in Table 1.

Table 1. Types and models of sensors used in the e-nose prototype.

\begin{tabular}{|c|c|c|}
\hline Sensor number & Model & Detected substances \\
\hline $\mathrm{S}_{1}$ & MiniPID & volatile organic compounds \\
\hline $\mathrm{S}_{2}$ & FECS44-100 & ammonia, amines \\
\hline $\mathrm{S}_{3}$ & FECS50-100 & hydrogen sulfide \\
\hline $\mathrm{S}_{4}$ & TGS2600 & air pollutions \\
\hline $\mathrm{S}_{5}$ & TGS2602 & volatile organic compounds \\
\hline $\mathrm{S}_{6}$ & TGS2603 & air pollutions (triethylamine, mercaptanes) \\
\hline $\mathrm{S}_{7}$ & TGS823 & organic solvent vapours \\
\hline $\mathrm{S}_{8}$ & TGS8100 & hydrogen, ethanol \\
\hline
\end{tabular}

\section{Experimental}

A group of probants took part in the research, who were to evaluate the prepared samples in terms of odour intensity. The research team consisted of 6 people who were familiarized with the guidelines concerning sensory tests. They assessed each of he prepared samples using a seven-grade verbal scale: 0 - not perceptible, 1 - very weak, 2 - weak, 3 - distinct, 4 - strong, 5 - very strong, 6 - extremely strong.

Five samples of trimethylamine and trimethylamine were prepared in deionized water at the following concentration levels: 50,100, 200, 400 and $800 \mathrm{ppm} \mathrm{v/v.} \mathrm{The} \mathrm{samples} \mathrm{were}$ subjected to sensory analysis in terms of the odour intensity. A group of six probants performed the odour evaluation using a verbal-point scale, each measurement was repeated five times for each person and the concentration level. The average values of the odour intensity for aqueous solutions of the analyzed substances are presented in the Table 2.

Table 2. The average values of the odour intensity of trimethylamine (TMA) and triethylamine (TEA) aqueous solutions.

\begin{tabular}{|c|c|c|}
\hline \multirow{2}{*}{ Concentration, $\mathbf{p p m} \mathbf{~ v / v}$} & \multicolumn{2}{|c|}{ Average odour intensity (sensory analysis) } \\
\cline { 2 - 3 } & trimethylamine & triethylamine \\
\hline 50 & 0.8 & 0.2 \\
\hline 100 & 1.4 & 0.9 \\
\hline 200 & 2.4 & 1.6 \\
\hline 400 & 2.8 & 2.5 \\
\hline 800 & 3.6 & 3.2 \\
\hline
\end{tabular}

Samples with the same concentrations were tested using an electronic nose prototype. Three analyzes were performed for each substance and concentration. To develop the 
calibration model, the maximal value of the sensor's signal after the exposition on a given sample was used. An example of the MOS sensor response is shown in the Figure 2.

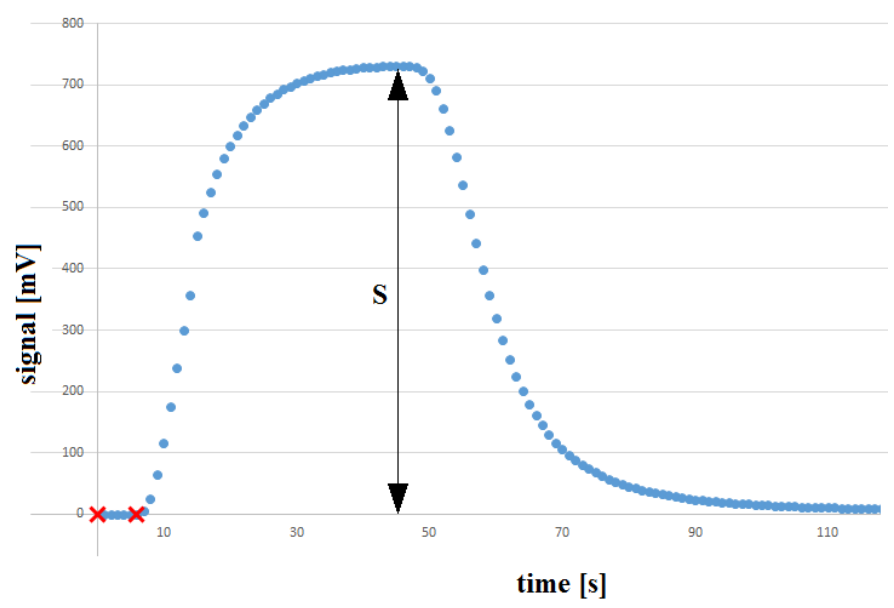

Fig. 2. MOS sensor signal (the interval between the red crosses indicates the baseline).

On the basis of the obtained signals using a multiparameter linear regression (MLR), an equation was determined that links the fragrance intensity of the sample to the signal from individual sensors [21]. After estimating the confidence interval of the model parameters statistically non-significant model parameters were removed from the equation. The equation takes the form of (6):

$$
I=12.7574-0.0143 \cdot S_{2}-0.0142 \cdot S_{4}+0.0118 \cdot S_{6}+0.0016 \cdot S_{8}
$$

\section{Results and discussion}

Twenty five binary mixtures of TMA and TEA were prepared. Next, the theoretical values of the fragrance intensity with the use of Zwaardemaker $\left(\mathrm{I}_{\mathrm{Zw}}\right)$ and EA ( $\left.\mathrm{I}_{\mathrm{EA}}\right)$ models were determined for them. For each sample an electronic nose analysis was also performed. The odour intensity was determined using the model (6). The obtained results are presented in the Table 3.

Analyzing the results presented in Table 4, it can be concluded that in the case of studied mixtures Zwaardemaker model and the EA model correlate to each other, giving similar results. However, it should be noted that in the case of high concentrations of the amines (trimethylamine and triethylamine) in the mixture, the difference between the results obtained using e-nose with MLR model and theoretical models are greater than $10 \%$ (relative error related to EA and Zwaardemaker model). This fact indicates the presence of odour interactions in the tested mixtures. In addition, all these values are negative. This indicates the occurrence of masking effect. 
Table 3. Odour intensity values determined using Zwaardemaker model, EA model and using electronic nose.

\begin{tabular}{|c|c|c|c|c|c|c|}
\hline \multicolumn{2}{|c|}{ Mixture composition } & \multirow[b]{2}{*}{$\mathbf{I}_{\mathbf{Z w}}$} & \multirow[b]{2}{*}{$\mathbf{I}_{\mathbf{E A}}$} & \multirow[b]{2}{*}{$\mathbf{I}_{\mathrm{e}-\text { nose }}$} & \multirow{2}{*}{$\begin{array}{c}\text { Error } \\
\frac{I_{E A}-I_{e-\text { nose }}}{I_{E A}}\end{array}$} & \multirow{2}{*}{$\begin{array}{c}\text { Error } \\
\frac{I_{Z w}-I_{e-\text { nose }}}{I_{Z w}}\end{array}$} \\
\hline $\mathrm{C}_{\text {TMA, }} \mathbf{p p m} \mathrm{v} / \mathrm{v}$ & $\mathrm{C}_{\text {TEA, }}$ ppm v/v & & & & & \\
\hline 50 & 50 & 0.84 & 0.82 & 0.80 & $-2 \%$ & $-5 \%$ \\
\hline 50 & 75 & 1.07 & 1.00 & 1.05 & $5 \%$ & $-2 \%$ \\
\hline 50 & 100 & 1.30 & 1.22 & 1.31 & $8 \%$ & $1 \%$ \\
\hline 50 & 150 & 1.67 & 1.58 & 1.66 & $5 \%$ & $-1 \%$ \\
\hline 50 & 200 & 1.95 & 1.85 & 1.91 & $3 \%$ & $-2 \%$ \\
\hline 75 & 50 & 1.25 & 1.23 & 1.21 & $-1 \%$ & $-3 \%$ \\
\hline 75 & 75 & 1.43 & 1.36 & 1.31 & $-3 \%$ & $-8 \%$ \\
\hline 75 & 100 & 1.62 & 1.52 & 1.55 & $2 \%$ & $-4 \%$ \\
\hline 75 & 150 & 1.94 & 1.82 & 1.87 & $3 \%$ & $-4 \%$ \\
\hline 75 & 200 & 2.20 & 2.07 & 2.25 & $9 \%$ & $2 \%$ \\
\hline 100 & 50 & 1.54 & 1.52 & 1.45 & $-4 \%$ & $-6 \%$ \\
\hline 100 & 75 & 1.70 & 1.62 & 1.72 & $6 \%$ & $1 \%$ \\
\hline 100 & 100 & 1.87 & 1.76 & 1.90 & $8 \%$ & $2 \%$ \\
\hline 100 & 150 & 2.16 & 2.03 & 2.05 & $1 \%$ & $-5 \%$ \\
\hline 100 & 200 & 2.40 & 2.25 & 1.76 & $-22 \%$ & $-27 \%$ \\
\hline 150 & 50 & 1.95 & 1.93 & 2.00 & $4 \%$ & $3 \%$ \\
\hline 150 & 75 & 2.09 & 2.01 & 2.06 & $2 \%$ & $-1 \%$ \\
\hline 150 & 100 & 2.24 & 2.12 & 2.07 & $-3 \%$ & $-8 \%$ \\
\hline 150 & 150 & 2.50 & 2.35 & 1.83 & $-22 \%$ & $-27 \%$ \\
\hline 150 & 200 & 2.71 & 2.54 & 2.09 & $-18 \%$ & $-23 \%$ \\
\hline 200 & 50 & 2.24 & 2.22 & 2.36 & $7 \%$ & $6 \%$ \\
\hline 200 & 75 & 2.37 & 2.29 & 2.35 & $3 \%$ & $-1 \%$ \\
\hline 200 & 100 & 2.51 & 2.39 & 2.57 & $7 \%$ & $2 \%$ \\
\hline 200 & 150 & 2.75 & 2.59 & 2.21 & $-15 \%$ & $-20 \%$ \\
\hline 200 & 200 & 2.95 & 2.77 & 2.38 & $-14 \%$ & $-19 \%$ \\
\hline
\end{tabular}

\section{Conclusions}

The obtained results presents that electronic noses can be successfully used to estimate the fragrance intensity of air samples especially in areas where amine compounds are responsible for the occurrence of odour nuisances. Research conducted on binary odour compounds mixtures shows that simple theoretical models do not actually reflect all interactions occurring in the studied samples. Conformity of results was obtained only for low concentrations of substances in the mixture $(<150 \mathrm{ppm} \mathrm{v} / \mathrm{v})$. This is due to the limitation of models, especially the EA model, which does not take into account the interaction coefficient at all. Only two-component mixtures were used in the tests. In further studies, it is planned to compare the results with 3-5 component mixtures. This approach determines the use of more sophisticated pattern recognition algorithms, i.e. artificial neural networks or fuzzy logic systems. However, as shown in the paper, for simple systems it is possible to use a MLR model with relatively high accuracy $\left(R^{2}=0.917\right)$.

In the conducted research, it was also shown that due to the convergence of results, the mechanisms of identifying fragrances implemented in the electronic nose are very similar to the mechanisms occurring in the human sense of smell. Additional advantages of using e-noses are the ability to omit the initial sample preparation stage, as well as the ability to work in the field and carry out measurements in the on-line mode. It is possible to optimize 
the number and type of sensors used to detect specific substances, which will allow to create devices dedicated to a specific application. Dynamic progress in the field of analytical devices from the group of electronic noses is expected to contribute to the development of odours analysis.

The investigations were financially supported by the Grant No. UMO-2015/19/B/ST4/02722 from the National Science Centre, Poland.

\section{References}

1. J. W. Gardner, P. N. Bartlett, Sens. Actuators B 18, 211-220 (1994)

2. P. Boeker, Sens. Actuators B 204, 2-17 (2014)

3. J. Gębicki, Trends Anal. Chem. 77, 1-13 (2016)

4. R. M. Stuetz, R. A. Fenner, G. Engin, Water Res. 33, 442-452 (1999)

5. S. Deshmukh, R. Bandyopadhyay, N. Bhattacharyya, R. A. Pandey, A. Jana, Talanta 144, 329-340 (2015)

6. W. Cynkar, D. Cozzolino, B. Dambergs, L. Janik, M. Gishen, Sens. Actuators B 124, 167-171 (2007)

7. T. Alizadeh, S. Zeynali, Sens. Actuators B 129, 412-423 (2008)

8. L. Spinelle, M. Gerboles, G. Kok, S. Persijn, T. Sauerwald, Sensors 17, 1520 (2017)

9. B. Szulczyński, J. Gębicki, Environments 4, 21, (2017)

10. D. T. Hill, C. L. Barth, Trans. of ASAE 19, 939-944 (1976)

11. T. C. Pearce, J. W. Gardner, Analyst 123, 2057-2066 (1998)

12. J. Kośmider, Archiwum Ochrony Środowiska 29, 17-30 (2003)

13. L. Yan, J. Liu, S. Jiang, Ch. Wu, K. Gao, Sensors 17, 1624 (2017)

14. Z. Wei, J. Wang, W. Zhang, Food Chem. 177, 89-96 (2015)

15. A. Loutfi, S. Coradeschi, G. K. Mani, P. Shankar, J. S. B. Rayappan, J. Food Eng. 144, 103-111(2015)

16. J. Gebicki, B. Szulczyński, M. Kaminski, Meas. Sci. Technol. 26, 125103 (2015)

17. R. López, I. O. Cabeza, I. Giráldez, M. J. Díaz, Bioresour. Technol. 102, 7984-7993 (2011)

18. B. Szulczyński, K. Armiński, J. Namieśnik, J. Gębicki, Sensors 18, 519 (2018)

19. B. Szulczyński, J. Gębicki, J. Namieśnik, Chem. Pap. 72, 527-532 (2018)

20. M. Delgado-Rodríguez, M. Ruiz-Montoya, I. Giraldez, R. López, E. Madejón, M. J. Díaz, Atmos. Environ. 51, 278-285 (2012)

21. B. Szulczyński, P. Rybarczyk, J. Gębicki, Monatsh. Chem. 149, 1693-1699 (2018)

22. J. H. Sohn, M. Dunlop, N. Hudson, T. I. Kim, Y. H. Yoo, Sens. Actuators B 135, 455-464 (2009)

23. B. Szulczyński, J. Namieśnik, J. Gębicki, Sensors 17, 2380 (2017) 